TRANSACTIONS OF THE

AMERICAN MATHEMATICAL SOCIETY

Volume 355, Number 12, Pages 4723-4735

S 0002-9947(03)03085-X

Article electronically published on July 24, 2003

\title{
THE CENTRAL LIMIT PROBLEM FOR CONVEX BODIES
}

\author{
MILLA ANTTILA, KEITH BALL, AND IRINI PERISSINAKI
}

\begin{abstract}
It is shown that every symmetric convex body which satisfies a kind of weak law of large numbers has the property that almost all its marginal distributions are approximately Gaussian. Several quite broad classes of bodies are shown to satisfy the condition.
\end{abstract}

\section{INTRODUCTION}

This paper describes a central limit theorem for symmetric, convex bodies. Let $K$ be a symmetric, convex body of volume 1 . We regard $K$ as a probability space and in each direction $\theta$, we define the random variable $X_{\theta}: x \mapsto\langle x, \theta\rangle$ : so the density of $X_{\theta}$ is obtained by scanning across $K$ with hyperplanes perpendicular to $\theta$. Now suppose that $K$ is isotropic, i.e. that for some fixed $\rho$

$$
\int_{K}\langle x, \theta\rangle^{2} d x=\rho^{2} \quad \text { for all } \theta .
$$

(We remark that each $K$ has an affine image which is isotropic.) Then each of the random variables $X_{\theta}$ has variance $\rho^{2}$.

Our aim is to show that most of these r.v.s are very close to a Gaussian r.v. $\gamma$ with variance $\rho^{2}$. We shall prove this under the following hypothesis, which states that the Euclidean norm concentrates near the value $\sqrt{n} \rho$, as a function on $K$.

Concentration Hypothesis. For a given $\varepsilon<\frac{1}{2}$ we say that $K$ satisfies the $\varepsilon$ concentration hypothesis if

$$
P\left(\left|\frac{|x|}{\sqrt{n}}-\rho\right| \geq \varepsilon \rho\right) \leq \varepsilon .
$$

Under the above hypothesis, we shall show that if $\delta>0$, then except for a set of directions of spherical measure at most $n e^{-\frac{n \delta^{2}}{50}}$, for every positive $t$, the probability $P\left(\left|X_{\theta}\right|<t\right)$ differs from $P(|\gamma|<t)$ by at most

$$
\delta+4 \varepsilon+\frac{c}{\sqrt{n}}
$$

At first sight, the concentration hypothesis (1) looks, at once, too strong to be true, except in trivial cases, and too weak to be useful. On the one hand, an estimate like (1), with a small value of $\varepsilon$, is considerably stronger than the estimate which follows from Borell's inequality or its recent generalisations and sharpenings

Received by the editors July 14, 1999.

2000 Mathematics Subject Classification. Primary 52A22; Secondary 60F05.

The first author was supported by EPSRC-97409672, and the second author was supported in part by NSF grant DMS-9257020. 
[7. On the other hand, although (11) states that most of $K$ lies in a thin spherical shell, $K$ will almost always occupy only a miniscule fraction, $e^{-n}$, of this shell. So the condition cannot automatically guarantee that $K$ "looks like" a spherical shell.

We deal with the second point by using standard concentration methods on the sphere together with a Lipschitz estimate that depends ultimately upon a version of the Brunn-Minkowski inequality. Concerning the first point, we believe that all isotropic convex bodies will satisfy (1) with $\varepsilon$ no more than about

$$
\frac{\log n}{\sqrt{n}} .
$$

In the last two sections we prove (11) with a small $\varepsilon$ for two classes of bodies which together include a significant portion of the space of convex bodies. The first is the class of $l_{p}^{n}$ balls, for which we obtain (1) with $\varepsilon \approx \frac{1}{n^{1 / 3}}$. To prove the concentration property we use the subindependence of complements of coordinate slabs in the $l_{p}^{n}$ ball, which was proved in [4. The second class is somewhat ad hoc, consisting of uniformly convex bodies which have the additional property of being contained in a Euclidean ball of appropriate radius, the necessary radius being dependent upon the modulus of convexity of $K$. However, this class encompasses all $l_{p}^{n}$ balls for $1<p<\infty$; so it is fairly broad.

In the ensuing discussion we have chosen, for the sake of clarity, to separate the abstract part, which holds for all convex, symmetric bodies under the concentration hypothesis (11), from the proofs of the hypothesis for specific bodies.

We should remark that the above results are very much in the same spirit as some results of Diaconis and Freedman [5], Sudakov [10] and von Weizsäcker [11. These show, in a general probabilistic setting, that a kind of weak law of large numbers implies that most marginals are approximately Gaussian under certain conditions. In the case of convex bodies we expect to obtain, and indeed do achieve, much finer probability estimates than for the general case.

\section{The abStract ARgument}

Let us denote by $g_{\theta}(s)$ the density of the r.v. $X_{\theta}: x \mapsto\langle x, \theta\rangle$ and by $g(s)$ that of the Gaussian r.v. $\gamma$, with variance $\rho^{2}$. The probability that $X_{\theta}$ is less than a value $t$ is the volume of $K$ to one side of the slice $K \cap\left(\langle\theta\rangle^{\perp}+t \theta\right)$. This volume can be expressed as an integral of volumes of the $(n-1)$-dimensional parallel slices $K \cap\left(\langle\theta\rangle^{\perp}+s \theta\right)$, where $s$ runs from $-\infty$ to $t$. Hence

$$
g_{\theta}(s)=\operatorname{vol}_{n-1}\left(K \cap\left(\langle\theta\rangle^{\perp}+s \theta\right)\right) .
$$

Obviously,

$$
g(s)=\frac{1}{\rho \sqrt{2 \pi}} \exp \left(-\frac{s^{2}}{2 \rho^{2}}\right) .
$$

So, in terms of integrals of densities, our aim is to show that for $\delta>0$

$$
\sigma\left(\left\{\theta:\left|\int_{-t}^{t} g_{\theta}(s) d s-\int_{-t}^{t} g(s) d s\right| \leq \delta+4 \varepsilon+\frac{c}{\sqrt{n}} \quad \text { for all } t\right\}\right) \geq 1-n e^{-\frac{n \delta^{2}}{50}}
$$

under the concentration hypothesis (10). (Here $\sigma$ denotes the rotation-invariant probability measure on the sphere $S^{n-1}=\left\{x \in \mathbf{R}^{n}: \sum x_{i}^{2}=1\right\}$.) 
The proof is composed of three main steps. We begin by considering not the individual $X_{\theta}$ and $g_{\theta}$, but an average of $g_{\theta}$ over all $\theta$ :

$$
A(t)=\int_{S^{n-1}} \int_{-t}^{t} g_{\theta}(s) d s d \sigma
$$

This averaging enables us to ignore how the volume of $K$ is distributed within the relevant spherical shell. We approximate $A(t)$ by an integral over $K$, of functions that are densities of Gaussian random variables of different variances. The concentration hypothesis is then used to show that most of the Gaussians have about the same variance. This will ensure that

$$
\left|A(t)-\int_{-t}^{t} g(s) d s\right| \leq 4 \varepsilon+\frac{c_{1}}{\sqrt{n}} .
$$

Then, using standard concentration of measure results and the fact that $\int_{-t}^{t} g_{\theta}(s) d s$ is the reciprocal of a norm, we get that for each $t$

$$
\sigma\left(\left\{\theta:\left|\int_{-t}^{t} g_{\theta}(s) d s-\int_{-t}^{t} g(s) d s\right| \geq \delta+4 \varepsilon+\frac{c_{3}}{\sqrt{n}}\right\}\right) \leq 2 e^{-\frac{n \delta^{2}}{50}}
$$

for each positive $\delta$.

Finally, by dividing the real line into intervals of appropriate width and using the Lipschitz property of the function

$$
H(t)=\left|\int_{-t}^{t} g_{\theta}(s) d s-\int_{-t}^{t} g(s) d s\right|
$$

we obtain a result for every $t$ simultaneously.

Our first theorem estimates the spherical average $A(t)$ of the probability $P\left(\left|X_{\theta}\right| \leq t\right)$ :

Theorem 1. Under the concentration hypothesis (1), for all positive numbers $t$,

$$
\left|A(t)-\int_{-t}^{t} g(s) d s\right| \leq 4 \varepsilon+\frac{c_{1}}{\sqrt{n}}
$$

We begin with a simple geometric lemma which approximates the average $A(t)$ by an integral over $K$.

\section{Lemma 1.}

$$
\left|A(t)-\frac{2}{\sqrt{2 \pi}} \int_{K} \int_{0}^{\frac{t \sqrt{n}}{|x|}} e^{-\frac{v^{2}}{2}} d v d x\right| \leq \frac{c_{1}}{\sqrt{n}} .
$$

Proof. If $v$ is a unit vector in $\mathbf{R}^{n}$, then

$$
\sigma(\{\theta:|\langle\theta, v\rangle|<t\})=\frac{\int_{0}^{t}\left(1-u^{2}\right)^{\frac{n-3}{2}} d u}{\int_{0}^{1}\left(1-u^{2}\right)^{\frac{n-3}{2}} d u} .
$$


We then have

$$
\begin{aligned}
\int_{S^{n-1}} \int_{-t}^{t} g_{\theta}(s) d s d \sigma & =\int_{S^{n-1}} \int_{-t}^{t} \operatorname{vol}_{n-1}\left(K \cap\left(\langle\theta\rangle^{\perp}+s \theta\right)\right) d s d \sigma(\theta) \\
& =\int_{S_{n-1}} \int_{K} \mathbf{1}_{\{-t \leq\langle x, \theta\rangle \leq t\}} d x d \sigma(\theta) \\
& =\int_{K} \sigma\left(\left\{-\frac{t}{|x|} \leq\left\langle\frac{x}{|x|}, \theta\right\rangle \leq \frac{t}{|x|}\right\}\right) d x \\
& =\int_{K} \frac{\int_{0}^{\frac{t}{x \mid}}\left(1-u^{2}\right)^{\frac{n-3}{2}} d u}{\int_{0}^{1}\left(1-u^{2}\right)^{\frac{n-3}{2}} d u} d x
\end{aligned}
$$

It is not hard to check that the integrand differs from the integral of the Gaussian by at most a constant over $\sqrt{n}$. Since the volume of $K$ is 1 , the lemma follows.

From now on, let

$$
F(s)=\frac{2}{\sqrt{2 \pi}} \int_{0}^{\frac{t}{s}} e^{-\frac{v^{2}}{2}} d v
$$

denote the integral of the Gaussian density with variance $s^{2}$. To prove Theorem 1 we need to show that the average $\int_{K} F\left(\frac{|x|}{\sqrt{n}}\right) d x$ is close to $F(\rho)$. Here we invoke the concentration hypothesis.

We divide $K$ into two subsets: $K_{1}$, where $\frac{|x|}{\sqrt{n}}$ is within $\varepsilon \rho$ of $\rho$, and its complement in $K, K_{2}$. Since we find $F(s)$ to be Lipschitz with constant $\frac{2}{\rho}$ near $\rho, F\left(\frac{|x|}{\sqrt{n}}\right)$ is within $2 \varepsilon$ of $F(\rho)$ in $K_{1}$. The volume of $K_{2}$ is sufficiently small for it not to matter how far apart the functions are here.

Proof of Theorem 1. Let

$$
\begin{aligned}
& K_{1}=K \cap\left\{\left|\frac{|x|}{\sqrt{n}}-\rho\right| \leq \varepsilon \rho\right\}, \\
& K_{2}=K \cap\left\{\left|\frac{|x|}{\sqrt{n}}-\rho\right| \geq \varepsilon \rho\right\} .
\end{aligned}
$$

Then

$$
\begin{aligned}
& \left|\int_{K} F\left(\frac{|x|}{\sqrt{n}}\right) d x-F(\rho)\right| \\
& \leq \int_{K_{1}}\left|F\left(\frac{|x|}{\sqrt{n}}\right)-F(\rho)\right| d x+\int_{K_{2}}\left|F\left(\frac{|x|}{\sqrt{n}}\right)-F(\rho)\right| d x .
\end{aligned}
$$

To estimate the second integral, we need only recall that $F\left(\frac{|x|}{\sqrt{n}}\right)$ and $F(\rho)$ are at most one. Therefore, by the concentration hypothesis,

$$
\int_{K_{2}}\left|F\left(\frac{|x|}{\sqrt{n}}\right)-F(\rho)\right| \leq 2\left|K_{2}\right| \leq 2 \varepsilon .
$$

For the first integral we shall use a Lipschitz estimate for $F$. The derivative, $F^{\prime}(s)$, is bounded by $\frac{1}{s}$, so, provided $s>\frac{\rho}{2}$, we have a bound of order $\rho^{-1}$. In $K_{1}$ 
we have $\frac{|x|}{\sqrt{n}}>\frac{\rho}{2}$ since $\varepsilon<\frac{1}{2}$, and therefore

$$
\int_{K_{1}}\left|F\left(\frac{|x|}{\sqrt{n}}\right)-F(\rho)\right| d x \leq \int_{K_{1}} \frac{2}{\rho}\left|\frac{|x|}{\sqrt{n}}-\rho\right| d x \leq \int_{K_{1}} 2 \varepsilon d x \leq 2 \varepsilon .
$$

So we have

$$
\left|\int_{K} F\left(\frac{|x|}{\sqrt{n}}\right) d x-F(\rho)\right| \leq 4 \varepsilon .
$$

Combining this with Lemma 1, we get

$$
\left|A(t)-\int_{-t}^{t} g(s) d s\right| \leq 4 \varepsilon+\frac{c_{1}}{\sqrt{n}} .
$$

The problem is now to pass from an estimate for the average $A(t)$ to an estimate for specific directions.

Theorem 2. Under the concentration hypothesis (11), for each positive $t$ and $\delta$,

$$
\sigma\left(\left\{\theta:\left|\int_{-t}^{t} g_{\theta}(s) d s-\int_{-t}^{t} g(s) d s\right| \geq \delta+4 \varepsilon+\frac{c_{3}}{\sqrt{n}}\right\}\right) \leq 2 e^{-\frac{n \delta^{2}}{50}} .
$$

Central to the proof of Theorem 2 is a standard concentration of measure result of the type studied by Milman and others, based upon Levy's isoperimetric inequality on the sphere. For simple expositions of this kind of result, see [2] or [9].

Lemma 2. If $f: S^{n-1} \rightarrow \mathbf{R}$ is 1-Lipschitz and $M$ is its mean, then

$$
\sigma\left(\left\{\theta:|f-M| \geq \delta+\frac{c_{2}}{\sqrt{n}}\right\}\right) \leq 2 e^{-\frac{n \delta^{2}}{2}} .
$$

Obviously we shall take $\int_{-t}^{t} g_{\theta}(s) d s$ to be our Lipschitz function of $\theta$, and so our $M$ will be $A(t)$, which we already know from Theorem 1 to be close to the required $\int_{-t}^{t} g(s) d s$. To obtain the Lipschitz estimate we shall show that $\int_{-t}^{t} g_{\theta}(s) d s$ is the reciprocal of a norm (restricted to $S^{n-1}$ ). To do this, we apply Busemann's theorem to a certain convex body in $\mathbf{R}^{n+1}$ constructed from $K$.

Theorem 3 (Busemann's theorem). Let $C$ be a symmetric convex body in $\mathbf{R}^{n}$, and for each unit vector $u$ let $r(u)$ be the volume of the slice of $C$ by the subspace orthogonal to $u$. Then the body whose radius in each direction $u$ is $r(u)$ is itself convex.

Notice that, as a consequence of Busemann's theorem, $\frac{1}{r(u)}$ is a restriction to the sphere of a norm. Lemma 3 below is closely related to the so-called "convexity of the floating body", which was proved simultaneously by Meyer and Reisner [8] and by the second-named author. We shall use the latter's argument, but the difference here is that the earlier proofs involved slabs of fixed volume, whereas here we fix the slab width.

Lemma 3. For all positive $t$,

$$
\|x\|=\frac{|x|}{\int_{-t}^{t} g_{\frac{x}{|x|}}(s) d s}
$$

defines a norm on $\mathbf{R}^{n}$. 
Proof. Let us first recall that $g_{\frac{x}{|x|}}(s)=\left|K \cap\left(\frac{x}{|x|}{ }^{\perp}+s \frac{x}{|x|}\right)\right|$. We will denote the volume of the slab of $K$ perpendicular to $x$ and of width $2 t$ by

$$
v(x, t)=\int_{-t}^{t} g_{\frac{x}{|x|}}(s) d s .
$$

Our aim is thus to prove the following triangle inequality for all $x, y \in \mathbf{R}^{n}$ :

$$
\frac{1}{2}\left(\frac{|x|}{v(x, t)}+\frac{|y|}{v(y, t)}\right) \geq \frac{\left|\frac{x+y}{2}\right|}{v\left(\frac{x+y}{2}, t\right)} .
$$

Notice that the result is obvious when the angle between $x$ and $y$ is zero or $\pi$.

We consider the convex body

$$
K^{\prime}=K \times[-1,1] \quad \subset \quad \mathbf{R}^{n} \times \mathbf{R}=\mathbf{R}^{n+1} .
$$

Busemann's theorem tells us that, since $K^{\prime}$ is a symmetric, convex body in $\mathbf{R}^{n+1}$, then $\frac{|\theta|}{\left|K^{\prime} \cap \theta^{-}\right|}$defines a norm on $\mathbf{R}^{n+1} /\{0\}$. Hence

$$
\frac{1}{2}\left(\frac{|\theta|}{\left|K^{\prime} \cap \theta^{\perp}\right|}+\frac{|\phi|}{\left|K^{\prime} \cap \phi^{\perp}\right|}\right) \geq \frac{\left|\frac{\theta+\phi}{2}\right|}{\left|K^{\prime} \cap\left(\frac{\theta+\phi}{2}\right)^{\perp}\right|}
$$

for all $\theta, \phi \in \mathbf{R}^{n+1}$.

Given $x \in \mathbf{R}^{n}$ and $t=\frac{\sqrt{1-r^{2}}}{r}$, where $r>0$, if we choose $u \in \mathbf{R}^{n+1}$ as

$$
u=\left(r \frac{x}{|x|}, \sqrt{1-r^{2}}\right),
$$

then the projection of $K^{\prime} \cap u^{\perp}$ onto the first $n$ coordinates is precisely the slab of $K$ perpendicular to $x$ and of width $2 t$. The ratio of $v(x, t)$ to the volume $\left|K^{\prime} \cap u^{\perp}\right|$ is then just $\sqrt{1-r^{2}}$. (Observe that $r$ determines the angle between $u^{\perp}$ and $\mathbf{R}^{n}$ ).

Now take $\theta=|x| u$ so that $\left|K^{\prime} \cap u^{\perp}\right|=\left|K^{\prime} \cap \theta^{\perp}\right|$ and $|\theta|=|x|$. Then inequality (2) simplifies to

$$
\frac{1}{2}\left(\frac{|x|}{v(x, t)}+\frac{|y|}{v(y, t)}\right) \geq \frac{1}{\sqrt{1-r^{2}}} \frac{\left|\frac{\theta+\phi}{2}\right|}{\left|K^{\prime} \cap\left(\frac{\theta+\phi}{2}\right)^{\perp}\right|},
$$

where we have repeated the above for $y \in \mathbf{R}^{n}$ with $\phi$.

Now

$$
\frac{\theta+\phi}{2}=\left(r \frac{(x+y)}{2}, \sqrt{1-r^{2}} \frac{(|x|+|y|)}{2}\right) .
$$

Hence the projection of $K^{\prime} \cap\left(\frac{\theta+\phi}{2}\right)^{\perp}$ is a slab perpendicular to $\frac{x+y}{2}$ whose width now depends on $r,\left|\frac{x+y}{2}\right|$ and $\frac{|x|+|y|}{2}$. In fact the width is $2 s$, where

$$
s=\frac{|x|+|y|}{|x+y|} t,
$$

and we get

$$
\frac{v\left(\frac{x+y}{2}, s\right)}{\left|K^{\prime} \cap\left(\frac{\theta+\phi}{2}\right)^{\perp}\right|}=\frac{\sqrt{1-r^{2}}}{2} \frac{(|x|+|y|)}{\left|\frac{\theta+\phi}{2}\right|} .
$$


Inequality (2) thus simplifies further to

$$
\frac{1}{2}\left(\frac{|x|}{v(x, t)}+\frac{|y|}{v(y, t)}\right) \geq \frac{1}{2} \frac{(|x|+|y|)}{v\left(\frac{x+y}{2}, s\right)} .
$$

Now we need only notice that for any $a \geq 1$ and $x \in R^{n}$ we have $v(x, a t) \leq a v(x, t)$, since $g_{\frac{x}{|x|}}(a s) \leq g_{\frac{x}{|x|}}(s)$ for all $s$. So

$$
\frac{1}{2} \frac{(|x|+|y|)}{v\left(\frac{x+y}{2}, s\right)} \geq \frac{\left|\frac{x+y}{2}\right|}{v\left(\frac{x+y}{2}, t\right)} .
$$

Since $\int_{-t}^{t} g_{\theta}(s) d s$ is the reciprocal of a norm, we can get a Lipschitz estimate for this function just by estimating its size. This we do in the following lemmas. The first is a well-known consequence of the Brunn-Minkowski inequality, which implies that $g_{\theta}$ is a $\log$-concave function. (See, e.g., [3].)

Lemma 4. For all positive $t$,

$$
\int_{t}^{\infty} g_{\theta}(s) d s \leq \frac{1}{2} e^{-2 g_{\theta}(0) t} .
$$

Lemma 5. For each $t$ there are constants $a$ and $b$ so that if

$$
\|x\|=\frac{|x|}{\int_{-t}^{t} g_{\frac{x}{|x|}}(s) d s}
$$

then

where $\frac{b}{a} \leq 5$ and $\frac{1}{a} \leq 1$.

$$
a|x| \leq|| x|| \leq b|x|,
$$

Proof. Again from well-known results on log-concave functions (which can also be found in [3]) we can relate the value $g_{\theta}(0)$ to the variance $\rho$ in the following way.

$$
\frac{1}{\sqrt{12} \rho} \leq g_{\theta}(0) \leq \frac{1}{\sqrt{2} \rho} \text {. }
$$

So using Lemma 4 we get

$$
\int_{-t}^{t} g_{\theta}(s) d s \leq \min \left\{2 t g_{\theta}(0), 1\right\} \leq \min \left\{\frac{\sqrt{2} t}{\rho}, 1\right\}
$$

and

$$
\begin{aligned}
\int_{-t}^{t} g_{\theta}(s) d s & =1-2 \int_{t}^{\infty} g_{\theta}(s) d s \\
& \geq 1-e^{-2 g_{\theta}(0) t} \\
& \geq 1-e^{-\frac{t}{\sqrt{3} \rho}} \\
& \geq \min \left\{\frac{t}{3 \rho}, \frac{1}{3 \sqrt{2}}\right\} .
\end{aligned}
$$

Therefore, taking

$$
a=\frac{1}{\min \left\{\frac{\sqrt{2} t}{\rho}, 1\right\}}, \quad b=\frac{1}{\min \left\{\frac{t}{3 \rho}, \frac{1}{3 \sqrt{2}}\right\}},
$$

we obtain the required bounds, whatever the value of $\frac{t}{\rho}$. 
We are now ready to obtain the estimate for specific directions.

Proof of Theorem [2. As was explained earlier, we need to determine a Lipschitz estimate for $\int_{-t}^{t} g_{\theta}(s) d s$, i.e., to find a constant $d$ such that

$$
\left|\int_{-t}^{t} g_{\theta}(s) d s-\int_{-t}^{t} g_{\phi}(s) d s\right| \leq d|\theta-\phi| \quad \text { for } \theta, \phi \in S^{n-1}
$$

Now, with the norm notation used above,

$$
\begin{aligned}
\left|\int_{-t}^{t} g_{\theta}(s) d s-\int_{-t}^{t} g_{\phi}(s) d s\right| & =\left|\frac{1}{\|\theta\|}-\frac{1}{\|\phi\|}\right| \\
& \leq \frac{\|\theta-\phi\|}{\|\theta\|\|\phi\|} \\
& \leq \frac{b}{a^{2}}|\theta-\phi| \\
& \leq 5|\theta-\phi|
\end{aligned}
$$

by Lemma 5

Coupled with this Lipschitz estimate, Lemma 2 immediately gives

$$
\sigma\left(\left\{\theta:\left|\int_{-t}^{t} g_{\theta}(s) d s-A(t)\right| \geq \delta+\frac{c_{2}}{\sqrt{n}}\right\}\right) \leq 2 \exp \left(-\frac{n \delta^{2}}{50}\right) .
$$

Combining this with Theorem 1 which estimates $A(t)$, we get

$$
\sigma\left(\left\{\theta:\left|\int_{-t}^{t} g_{\theta}(s) d s-\int_{-t}^{t} g(s) d s\right| \geq \delta+4 \varepsilon+\frac{c_{3}}{\sqrt{n}}\right\}\right) \leq 2 \exp \left(-\frac{n \delta^{2}}{50}\right),
$$

as required.

To finish this section, we pass from Theorem 2 to a statement which holds for every $t$ simultaneously. This then tells us that most $X_{\theta}$ are very close to a Gaussian r.v. with variance $\rho^{2}$.

For a given $\theta$, let

$$
H(t)=\left|\int_{-t}^{t} g_{\theta}(s) d s-\int_{-t}^{t} g(s) d s\right|
$$

be the error at position $t$.

We saw in the proof of Lemma 5 that $g_{\theta}(s)$ is bounded above by $\frac{1}{\sqrt{2} \rho}$, and we recall that $g(s)$ is the Gaussian density $\frac{1}{\rho \sqrt{2 \pi}} e^{-s^{2} / 2 \rho^{2}}$. Hence

$$
\left|H^{\prime}(t)\right| \leq \frac{\sqrt{2}}{\rho}+\frac{2}{\rho \sqrt{2 \pi}},
$$

so $H$ is Lipschitz with a constant like $\frac{1}{\rho}$.

By pinning $H$ down at appropriate points we can use the Lipschitz property to pin it down elsewhere as long as we allow an additional error. We need only consider points in $[0,2 \rho \log n]$, since we see, with the aid of Lemma 4, that $H$ is sufficiently small for all $t$ beyond this interval. Dividing this interval into $2 \sqrt{n} \log n$ smaller intervals of length $\frac{\rho}{\sqrt{n}}$ gives an additional error of $\left(\sqrt{2}+\frac{2}{\sqrt{2 \pi}}\right) \frac{1}{\sqrt{n}}$, which is absorbed by the earlier error terms. We thus give up a factor of $2 \sqrt{n} \log n$ in the probability. 
Theorem 4. Under the concentration hypothesis (11), for $\delta>0$ we have

$$
\begin{aligned}
\sigma\left(\left\{\theta: H(t) \leq \delta+4 \varepsilon+\frac{c}{\sqrt{n}} \text { for all } t\right\}\right) & \geq 1-4 \sqrt{n} \log n e^{-\frac{n \delta^{2}}{50}} \\
& \geq 1-n e^{-\frac{n \delta^{2}}{50}} .
\end{aligned}
$$

\section{THE CONCENTRATION PROPERTY FOR $l_{p}^{n}$ BALLS}

In this section we show that $l_{p}^{n}$ balls satisfy the concentration hypothesis (1) with $\varepsilon \approx \frac{1}{n^{1 / 3}}$.

The precise statement is the following, in which $\rho_{n}^{2}$ is the variance for the appropriate ball.

Theorem 5. If $P$ is the Lebesgue measure on the normalized $l_{p}^{n}$ ball, then for all positive numbers $r$,

$$
P\left(\left|\frac{|x|^{2}}{n}-\rho_{n}^{2}\right| \geq r\right) \leq \frac{35 \rho_{n}{ }^{4}}{n r^{2}} .
$$

The estimate depends upon a subindependence property for the complements of coordinate slabs in $K$. Much stronger concentration results could doubtless be proved by approximating the bodies by independent random variables, but the argument here is a good deal simpler, and for our purposes, a better decay rate is of little use. The subindependence property for $l_{p}^{n}$ balls was proved by the second and third named authors in [4. Here we offer a more succinct proof, alluded to in the aforementioned article.

Lemma 6 (Subindependence of complements of coordinate slabs). If the probability $P$ is normalised Lebesgue measure on one of the $l_{p}^{n}$ balls, say $K$, then for any sequence $t_{1}, \ldots, t_{n}$ of positive numbers,

$$
P\left(\bigcap_{i=1}^{n}\left\{\left|x_{i}\right| \geq t_{i}\right\}\right) \leq \prod_{i=1}^{n} P\left(\left\{\left|x_{i}\right| \geq t_{i}\right\}\right) .
$$

Proof. Plainly it is enough to prove that

$$
P\left(\bigcap_{i=1}^{n}\left\{\left|x_{i}\right| \geq t_{i}\right\}\right) \leq P\left(\left|x_{1}\right| \geq t_{1}\right) P\left(\bigcap_{i=2}^{n}\left\{\left|x_{i}\right| \geq t_{i}\right\}\right) .
$$

We first rewrite the above with $S=\bigcap_{i=2}^{n}\left\{\left|x_{i}\right| \geq t_{i}\right\}$ :

$$
\frac{\left|K \cap S \cap\left\{\left|x_{1}\right| \geq t_{1}\right\}\right|}{|K|} \leq \frac{\left|K \cap\left\{\left|x_{1}\right| \geq t_{1}\right\}\right|}{|K|} \frac{|K \cap S|}{|K|} .
$$

Now we use the obvious fact that if $f:[0,1] \rightarrow \mathbf{R}$ is increasing and satisfies

$$
\int_{0}^{1} f d \mu=\alpha \mu[0,1]
$$

for some positive measure $\mu$ and constant $\alpha>0$, then

$$
\int_{0}^{s} f d \mu \leq \alpha \mu[0, s] \quad \text { for all } s \in[0,1]
$$

We take

$$
f(u)=\frac{\left|K \cap S \cap\left\{\left|x_{1}\right|=1-u\right\}\right|}{\left|K \cap\left\{\left|x_{1}\right|=1-u\right\}\right|}
$$


and let

$$
g(u)=\frac{\left|K \cap\left\{\left|x_{1}\right|=1-u\right\}\right|}{|K|}
$$

be the density of our measure $\mu$. Then

$$
\begin{aligned}
\int_{0}^{1} f(u) d \mu & =\int_{0}^{1} \frac{\left|K \cap S \cap\left\{\left|x_{1}\right|=1-u\right\}\right|}{\left|K \cap\left\{\left|x_{1}\right|=1-u\right\}\right|} \frac{\left|K \cap\left\{\left|x_{1}\right|=1-u\right\}\right|}{|K|} d u \\
& =\frac{|K \cap S|}{|K|}
\end{aligned}
$$

and

$$
\int_{0}^{1-t} f(u) d \mu=\frac{\left|K \cap S \cap\left\{\left|x_{1}\right| \geq t\right\}\right|}{|K|} .
$$

Taking $\alpha=\frac{|K \cap S|}{|K|}$ and $s=1-t_{1}$ in (3) completes the proof.

The corollary below, an anti-correlation inequality for the coordinate random variables $X_{i}: x \rightarrow\left\langle x, e_{i}\right\rangle$, follows directly from Lemma 1 if we notice that

$$
\int_{K \cap\left\{x_{1} \geq 0, x_{2} \geq 0\right\}} x_{1}^{2} x_{2}^{2} \quad \text { can be expressed as } 4 \int_{\mathbf{R}_{+}^{2}} u v P\left(x_{1} \geq u, x_{2} \geq v\right) d u d v .
$$

\section{Corollary 1.}

$$
\int_{K} x_{1}^{2} x_{2}^{2} \leq \int_{K} x_{1}^{2} \int_{K} x_{2}^{2}
$$

The proof of Theorem [5 relies on the fact that $\int_{K}|x|^{4}$ can be written in terms of $\sum_{1}^{n} \int_{K} x_{i}{ }^{4}$ and $\sum_{i \neq j} x_{i}{ }^{2} x_{j}{ }^{2}$. The second term is dealt with using subindependence. For the first we use a cruder estimate derived from a standard result concerning log-concave functions:

$$
\int_{K} x_{i}{ }^{4} \leq 36\left(\int_{K} x_{i}^{2}\right)^{2}
$$

The crudity doesn't matter, since there are so few contributions to this term.

Proof of Theorem [5. We first prove that $\frac{1}{n^{2}} \int_{K}|x|^{4}$ is close to $\rho_{n}^{4}$; namely, that

$$
\rho_{n}^{4} \leq \frac{1}{n^{2}} \int_{K}|x|^{4} \leq\left(1+\frac{35}{n}\right) \rho_{n}^{4}
$$

The first inequality is obvious by Cauchy-Schwarz.

For the second one we have

$$
\begin{aligned}
\int_{K}|x|^{4} & =\int_{K}\left(\sum_{i=1}^{n} x_{i}^{2}\right)^{2} \\
& =\sum_{1}^{n} \int_{K} x_{i}^{4}+\sum_{i \neq j} \int_{K} x_{i}^{2} x_{j}^{2} \\
& \leq n \int_{K} x_{i}^{4}+\sum_{i \neq j} \int_{K} x_{i}^{2} \int_{K} x_{j}^{2} \\
& \leq 36 n \rho_{n}^{4}+n(n-1) \rho_{n}^{4} \\
& =n^{2}\left(1+\frac{35}{n}\right) \rho_{n}^{4} .
\end{aligned}
$$


From this we can conclude that the integral $\int_{K}\left(\frac{|x|^{2}}{n}-\rho_{n}^{2}\right)^{2}$ is small and therefore that $\frac{|x|^{2}}{n}$ is close to $\rho_{n}^{2}$. Indeed,

$$
\begin{aligned}
0 \leq \int_{K}\left(\frac{|x|^{2}}{n}-\rho_{n}^{2}\right)^{2} & =\frac{1}{n^{2}} \int_{K}|x|^{4}-\frac{2 \rho_{n}^{2}}{n} \int_{K}|x|^{2}+\rho_{n}^{4} \\
& =\frac{1}{n^{2}} \int_{K}|x|^{4}-\frac{2}{n} \rho_{n}^{2} n \rho_{n}^{2}+\rho_{n}^{4} \\
& =\frac{1}{n^{2}} \int_{K}|x|^{4}-\rho_{n}^{4} \\
& \leq \frac{35}{n} \rho_{n}^{4} .
\end{aligned}
$$

So by Chebyshev's inequality we have

$$
\begin{aligned}
P\left(\left|\frac{|x|^{2}}{n}-\rho_{n}^{2}\right| \geq r\right) r^{2} & =P\left(\left(\frac{|x|^{2}}{n}-\rho_{n}^{2}\right)^{2} \geq r^{2}\right) r^{2} \\
& \leq \int_{K}\left(\frac{|x|^{2}}{n}-\rho_{n}^{2}\right)^{2} \\
& \leq \frac{35}{n} \rho_{n}^{4}
\end{aligned}
$$

By factoring the difference of two squares, we can estimate the deviation of $\frac{|x|}{\sqrt{n}}$ instead of $\frac{|x|^{2}}{n}$.

Corollary 2. For all positive numbers $u$,

$$
P\left(\left|\frac{|x|}{\sqrt{n}}-\rho_{n}\right| \geq u\right) \leq \frac{35 \rho_{n}^{2}}{n u^{2}} .
$$

By taking $u$ to be of the order of $\frac{1}{n^{1 / 3}}$ we obtain $\varepsilon$-concentration with $\varepsilon \approx \frac{1}{n^{1 / 3}}$.

\section{THE CONCENTRATION PROPERTY FOR UNIFORMLY CONVEX BODIES} CONTAINED IN SMALL EUCLIDEAN BALLS

$K$ is said to be uniformly convex if for every $\gamma>0$ we have

$$
\inf \left\{1-\frac{\|x+y\|}{2}:\|x\|,\|y\| \leq 1,\|x-y\| \geq \gamma\right\}=\delta(\gamma)>0
$$

where $\|$.$\| is the norm whose unit ball is K$. We shall consider bodies for which

$$
\delta(\gamma) \geq c \gamma^{q} \quad \text { for some } 2 \leq q<\infty,
$$

and assume that

$$
K \subset R \sqrt{n} B_{2}^{n} .
$$

Then we shall show that

Theorem 6. For $K$ satisfying (4) and (5),

$$
P\left(\left|\frac{|x|}{\sqrt{n}}-\rho\right|>\frac{c^{\prime} R \sqrt{\log n}}{n^{\frac{1}{q}}}\right)<\frac{1}{n} .
$$


Hence we have concentration as long as $\mu=\frac{c^{\prime} R \sqrt{\log n}}{n^{\frac{1}{q}}}$ is small. Notice that in order for $\mu$ to be small for a body with a good modulus of convexity, the Euclidean ball containing it may be quite large. As $K$ becomes less uniformly convex, it needs to be contained in a smaller ball.

Conditions (44) and (5) are satisfied in an appropriate way by all $l_{p}^{n}$ balls, for $1<p<\infty$. For $1<p \leq 2, \delta(\gamma) \approx(p-1) \gamma^{2}$. Here the bodies exhibit good uniform convexity, since the power of $\gamma$ remains 2. This compensates for the fact that $R=n^{\frac{1}{p}-\frac{1}{2}}$. On the other hand, for $2<p<\infty$, the uniform convexity deteriorates rapidly, since here $\delta(\gamma) \approx \gamma^{p}$. However, in this case $R$ is at most a constant.

Theorem [6] is a simple corollary of the following result of Gromov and Milman [6], which guarantees that uniformly convex bodies exhibit concentration with respect to the distance given by the norm. We use the hypothesis (5) to transfer the estimate to the Euclidean distance. The proof given here was found recently by J. Arias de Reyna, K. Ball and R. Villa [1].

Theorem 7 (Gromov-Milman). If $A \subset K$ has positive measure, and $d(x, A)$ is the distance from $x$ to $A$ (measured in the norm whose unit ball is $K$ ), then

$$
P(d(x, A)>\varepsilon)<\frac{e^{-2 n \delta(\varepsilon)}}{P(A)} .
$$

Proof. Suppose that $A, B \subset K$ are such that $B=\{x \in K: d(x, A)>\varepsilon\}$. Since $K$ is uniformly convex, if $x \in A$ and $y \in B$ then $\frac{\|x+y\|}{2} \leq 1-\delta(\varepsilon)$. Hence

$$
\frac{A+B}{2} \subset(1-\delta(\varepsilon)) K \text {. }
$$

By the Brunn-Minkowski inequality,

$$
P\left(\frac{A+B}{2}\right) \geq P(A)^{\frac{1}{2}} P(B)^{\frac{1}{2}}
$$

Combining (6) and (17), we get

$$
P(B) \leq \frac{(1-\delta(\varepsilon))^{2 n}}{P(A)} \leq \frac{e^{-2 n \delta(\varepsilon)}}{P(A)} .
$$

Proof of Theorem [6] Let $\lambda$ be the median of $\frac{|x|}{\sqrt{n}}$ on $K$ and $A=\left(\frac{|x|}{\sqrt{n}} \leq \lambda\right)$. Then, by Theorem 7

$$
P(d(x, A)>\gamma) \leq 2 e^{-2 n \delta(\gamma)}
$$

and

$$
P\left(d\left(x, A^{c}\right)>\gamma\right) \leq 2 e^{-2 n \delta(\gamma)} .
$$

Now if $y \in A$ and $d(x, y)<\gamma$, then $|x-y|<R \sqrt{n} \gamma$ since $K \subset R \sqrt{n} B_{2}^{n}$. Hence

$$
\begin{aligned}
P(|| x|-\lambda \sqrt{n}|>R \sqrt{n} \gamma) & \leq 4 e^{-2 n \delta(\gamma)} \\
& \leq 4 e^{-2 n c \gamma^{q}} .
\end{aligned}
$$

This implies that, for some constant $c^{\prime}$, the mean $\rho$ differs from the median $\lambda$ by at most $c^{\prime} R n^{-\frac{1}{q}}$. Hence

$$
P\left(\left|\frac{|x|}{\sqrt{n}}-\rho\right| \geq R \gamma+c^{\prime} R n^{-\frac{1}{q}}\right) \leq 4 e^{-2 c n \gamma^{q}}
$$

and, letting $\gamma=\frac{c^{\prime} \sqrt{\log n}}{n^{\frac{1}{q}}}$, we get the theorem. 


\section{REFERENCES}

[1] J.Arias de Reyna, K.Ball and R.Villa: Concentration of the distance in finite-dimensional normed spaces, Mathematika 45, 245-252, 1998. MR 2000b:46013

[2] K.Ball: An Elementary Introduction to Modern Convex Geometry, Flavors of Geometry, Mathematical Sciences Research Institute Publications 31, Cambridge Univ. Press, 1997, pp. 1-58. MR 99f:52002

[3] K.Ball: Logarithmically concave functions and sections of convex sets in $\mathbf{R}^{n}$, Stud. Math. 88, No.1, 69-84, 1988. MR 89e:52002

[4] K.Ball and I.Perissinaki: Subindependence of coordinate slabs in $l_{p}^{n}$ balls, Israel J. Math. 107, 289-299, 1998. MR 99k:52012

[5] P.Diaconis and D.Freedman: Asymptotics of graphical projection pursuit, Ann. of Stat. 12, 793-815, 1984. MR 86b:62011

[6] M. Gromov and V. Milman: Generalization of the spherical isoperimetric inequality to uniformly convex Banach spaces, Compos. Math. 62, 263-282, 1987. MR 89f:46031

[7] L.Lovász and M.Simonovits: Mixing rate of Markov chains, an isoperimetric inequality, and computing the volume, Proc. 31st Annual Symp. on Found. of Computer Science, IEEE Computer Soc., 346-355, 1990. MR 93e:68035

[8] M.Meyer and S.Reisner: A geometric property of the boundary of symmetric convex bodies and convexity of flotation surfaces, Geom Dedicata 37, No 3, 327-337, 1991 MR 92a:52003

[9] G. Pisier: The volume of convex bodies and Banach space geometry, Tracts in Math. 94, Cambridge Univ. Press, 1989. MR 91d:52005

[10] V. Sudakov: Typical distributions of linear functionals in finite-dimensional spaces of high dimension, Soviet Math. Dokl. 19 No 6, 1578-1582, 1978 MR 80b:60011

[11] H.von Weizsäcker: Sudakov's typical marginals, random linear functionals and a conditional central limit theorem, Probab. Theory Relat. Fields 107, 313-324, 1997. MR 99c:60006

Department of Mathematics, University of Kuopio, pl 1627, 70211 Kuopio, Finland

E-mail address: meanttila@hytti.uku.fi

Department of Mathematics, University College, University of London, Gower Street, London WC1E 6BT, England

E-mail address: kmb@math.ucl.ac.uk

Department of Mathematics, University of Crete, 710409 Iraklion, Greece

E-mail address: irinip@math.uoc.gr 